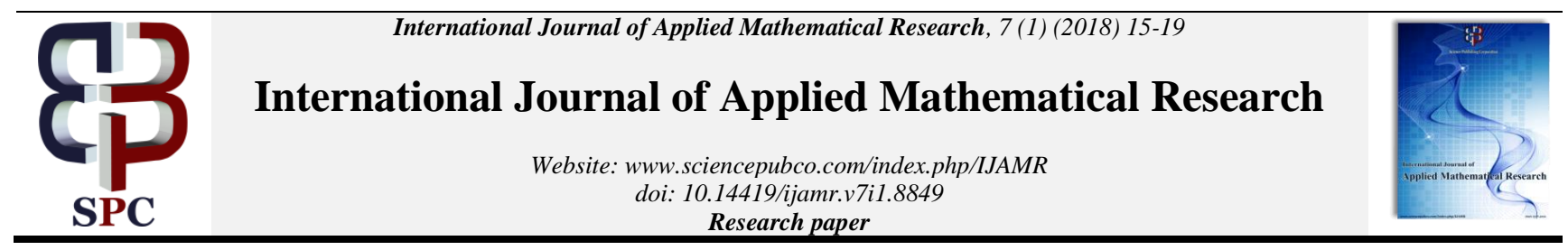

\title{
Analytical solutions for conformable fractional Bratu-type equations
}

\author{
Mousa Ilie 1, 2 , Jafar Biazar 1,3*, Zainab Ayati ${ }^{4}$ \\ ${ }^{1}$ Department of Mathematics, Guilan Science and Research Branch, Islamic Azad University, Rasht, Iran \\ ${ }^{2}$ Department of Mathematics, Rasht Branch, Islamic Azad University, Rasht, Iran \\ ${ }^{3}$ Department of Applied Mathematics, Faculty of Mathematical Sciences, University of Guilan, \\ P.O.Box.41335-1914, Guilan, Rasht, Iran \\ ${ }^{4}$ Department of Engineering sciences, Faculty of Technology and Engineering East of Guilan, \\ University of Guilan, P.C. 44891-63157, Rudsar-Vajargah, Iran \\ *Corresponding author E-mail: biazar@iaurasht.ac.ir
}

\begin{abstract}
Solving fractional differential equations have a prominent function in different science such as physics and engineering. Therefore, are different definitions of the fractional derivative presented in recent years. The aim of the current paper is to solve the fractional differential equation by a semi-analytical method based on conformable fractional derivative. Fractional Bratu-type equations have been solved by the method and to show its capabilities. The obtained results have been compared with the exact solution.
\end{abstract}

Keywords: Adomian Decomposition Method; Fractional Differential Equations; Conformable Fractional Derivative; Fractional Bratu-Type Equations.

\section{Introduction}

Solving fractional differential equations is very important but there are many fractional differential equations, which can't be solved analytically. Due to this fact, finding an approximate solution of fractional differential equations is clearly significant. In recent years, many effective methods have been proposed for finding approximate solution to fractional differential equations [3-13], such as Adomian decomposition method [14,15], homotopy perturbation method [16-19], homotopy analysis method [20], variational iteration method [21], generalized, differential transform method [22], finite difference method [23], semi-discrete scheme and Chebyshev collocation method [24], Wavelet Operational [25], Perturbation-iteration algorithm [26], and other methods [27-29]. Definitions of the fractional derivative presented in different forms such as Caputo, Liouville, and so on. Most recently a novel definition called comfortable fractional has been suggested to obtain fractional Derivative [1,2].]. The purpose of the paper is solving fractional equation by Adomian Decomposition method based on above-mentioned definition.

The organization of the paper is as follows: In Section 2, the basic definitions' amenable fractional derivative and conformable fractional integral are described. In section 3, extended Adomian decomposition method [30-33], based on conformable fractional derivative is described. In Section 4, the method is used to solve fractional Bratu-type equations with different initial value. Finally, discussions are given, in section 4.

\section{Basic definitions}

In this section some basic definitions and properties of the comfortable fractional derivative and integral have been explained.

\subsection{Conformable fractional derivative}

Given a function $f:[0, \infty) \rightarrow \mathbb{R}$. Then conformable fractional derivative of $f$ of order $\alpha$ is defined as follows

$$
T_{\alpha}(f)(x)=\lim _{\varepsilon \rightarrow 0} \frac{f\left(x+\varepsilon x^{1-\alpha}\right)-f(x)}{\varepsilon},
$$

For all $x>0, \alpha \in(0,1)$ [1]. If $f$ is $\alpha$-differentiable in some $(0, a), a>0$, and provided that $\lim _{x \rightarrow 0^{+}} \mathrm{T}_{\alpha}(f)(x)$ exists, then define $\mathrm{T}_{\alpha}(f)(0)=\lim _{x \rightarrow 0^{+}} \mathrm{T}_{\alpha}(f)(x)$.

If the conformable derivative of $f$ of order $\alpha$ exists, then we simply say that $f$ is $\alpha$-differentiable [1,2].

One can easily show that $\mathrm{T}_{\alpha}$ satisfies all the properties in the following properties [1]:

Let $\alpha \in(0,1]$ and $f$, and $g$ be $\alpha$-differentiable at a point $\mathrm{x}>0$, Then.

$$
\begin{aligned}
& \text { A. For } a, b \in \mathbb{R} \quad \mathrm{T}_{\alpha}(a f+b g)=a \mathrm{~T}_{\alpha}(f)+b \mathrm{~T}_{\alpha}(g), \\
& \text { B. For all } p \in \mathbb{R} \quad \mathrm{T}_{\alpha}\left(x^{p}\right)=p x^{p-\alpha}, \\
& \text { C. For all constant functions } f(x)=\lambda, \quad \mathrm{T}_{\alpha}(\lambda)=0 \text {, } \\
& \text { D. } \mathrm{T}_{\alpha}(f \cdot g)=g \cdot \mathrm{T}_{\alpha}(f)+f \cdot \mathrm{T}_{\alpha}(g), \\
& \text { E. } \mathrm{T}_{\alpha}\left(\frac{f}{g}\right)=\frac{g \cdot \mathrm{T}_{\alpha}(f)-f \cdot \mathrm{T}_{\alpha}(g)}{g^{2}} \\
& \text { F. } \mathrm{T}_{\alpha}(f)=x^{1-\alpha} \frac{d f}{d x} .
\end{aligned}
$$




\subsection{Conformable fractional integral}

Given a function $f:[a, \infty) \rightarrow \mathbb{R}, a \geq 0$. Then conformable fractional integral of $f$ is defined as the following

$$
I_{\alpha}^{a}(f)(x)=\int_{a}^{x} \frac{f(t)}{t^{1-\alpha}} d t
$$

Where the integral is the usual Riemann improper integral, and $\alpha \in(0,1)[1,2]$.

For simplicity, we have, $I_{\alpha}^{0}(f)(x)=I_{\alpha}(f)(x)$.

One of the well results is the following [1]:

For all $x \geq a$, and any continuous function in the domain of $\mathrm{I}_{\alpha}^{a}$, we have $\mathrm{T}_{\alpha}\left(\mathrm{I}_{\alpha}^{a} f(x)\right)=f(x)$.

\section{Modify adomian decomposition method based on conformable fractional derivative}

Consider a fractional nonlinear differential equation in the form

$T_{\alpha}^{n}(u)(x)-\mathcal{N}(u(x))=f(x)$,

$T_{\alpha}^{k}(u)(0)=c_{k}, k=0,1,2, \ldots, n-1$,

Where $T_{\alpha}^{n}=T_{\alpha} T_{\alpha} T_{\alpha} \ldots T_{\alpha}, \mathrm{n}$ time, $\mathcal{N}$ is a nonlinear operator, and $f(x)$ is a known analytic function.

It is assumed that the unknown function $u(x)$ can be expressed by an infinite series in the following form

$u(x)=\sum_{m=0}^{\infty} u_{m}(x)$

And the nonlinear term $\mathcal{N}(u)$ can be presented as an infinite series of polynomials, say

$\mathcal{N}(u)=\sum_{m=0}^{\infty} \mathcal{A}_{m}\left(u_{0}, u_{1}, \ldots, u_{m}\right)$,

Where $\mathcal{A}_{n}\left(u_{0}, u_{1}, \ldots, u_{n}\right)$ are called the Adomian polynomials and are defined by

$$
\mathrm{A}_{\mathrm{m}}=\frac{1}{\mathrm{~m} !}\left[\mathrm{T}_{\alpha}^{\mathrm{m}}\left[\mathrm{N}\left(\sum_{\mathrm{i}=0}^{\infty} \mathrm{u}_{\mathrm{i}}\left(\frac{1}{\alpha} \lambda^{\alpha}\right)^{\mathrm{i}}\right)\right]\right]_{\lambda=0}, \quad \mathrm{~m}=0,1,2, \ldots
$$

By applying the inverse operator,

$\left(T_{\alpha}^{n}\right)^{-1}=I_{\alpha}^{n}=I_{\alpha} I_{\alpha} \ldots I_{\alpha}=\int_{0}^{x} \int_{0}^{t_{1}} \ldots \int_{0}^{t_{n-1}}(.) d t_{n} d t_{n-1} \ldots d t_{1}$

On both sides of Eq. (3), the following equation has been derived,

$u(x)=\sum_{k=0}^{n-1} \frac{\left(T_{\alpha}^{k} u\right)(0)}{k !}\left(\frac{1}{\alpha} x^{\alpha}\right)^{k}+I_{\alpha}^{n}(f)+I_{\alpha}^{n}(\mathcal{N}(u(x))$.

Considering (4) and (5), we obtain

$\sum_{m=0}^{\infty} u_{m}=\sum_{k=0}^{n-1} \frac{\left(T_{\alpha}^{k} u\right)(0)}{k !}\left(\frac{1}{\alpha} x^{\alpha}\right)^{k}+I_{\alpha}^{n}(f)+$

$\sum_{m=0}^{\infty} I_{\alpha}^{n}\left(\mathcal{A}_{m}\left(u_{0}, u_{1}, \ldots, u_{m}\right)\right)$. (8)

Now, let us to define the following iterative equations, as Adomian scheme

$u_{0}=\sum_{k=0}^{n-1} \frac{\left(\mathrm{T}_{\alpha}^{k} u\right)(0)}{k !}\left(\frac{1}{\alpha} x^{\alpha}\right)^{k}+\mathrm{I}_{\alpha}^{n}(f)$,

$u_{m+1}=I_{\alpha}^{n}\left(\mathcal{A}_{m}\left(u_{0}, u_{1}, \ldots, u_{m}\right), \quad m=1,2, \ldots\right.$

If the equation (3) has an analytic solution, the series (4) would be Taylor expansion of fractional order, and one may recognize the exact solution, otherwise the partial sum

$u_{0}(x)+\sum_{m=1}^{M-1} u_{m}(x)$
Demonstrates the Mth-order approximation of the problem.

\section{Examples}

We solve fractional Bratu-type equation with different initial condition by the presented method in this section.

Example 1 Consider fractional Bratu-type equation with the following initial condition [15]

$\mathrm{T}_{\alpha}^{2}(u)(x)-2 e^{u(x)}=0, \quad 0<\alpha \leq 1,0 \leq x<1, \quad u(0)=$ $\mathrm{T}_{\alpha}(u)(0)=0$.

The exact solution of Eq. (11) is $u(x)=-2 \ln \left(\cos \left(\frac{1}{\alpha} x^{\alpha}\right)\right.$.

To solve this equation by ADM according to (7), we have

$u(x)=u(0)+\left(\mathrm{T}_{\alpha} u\right)(0)\left(\frac{1}{\alpha} x^{\alpha}\right)+\mathrm{I}_{\alpha}^{2}\left(2 e^{u(x)}\right)$.

By substituting (4) and (5) into (12), we derive:

$\sum_{m=0}^{\infty} u_{m}=0+\sum_{m=0}^{\infty} I_{\alpha}^{2}\left(\mathcal{A}_{m}\left(u_{0}, u_{1}, \ldots, u_{m}\right)\right.$

So, the solution by ADM consists of following scheme

$u_{0}(x)=0$,

$\mathrm{u}_{\mathrm{m}+1}(\mathrm{x})=\mathrm{I}_{\alpha}^{2}\left(\mathrm{~A}_{\mathrm{m}}\left(\mathrm{u}_{0}, \mathrm{u}_{1}, \ldots, \mathrm{u}_{\mathrm{m}}\right)\right)$,

Where

$\mathcal{A}_{m}\left(u_{0}, u_{1}, \ldots, u_{m}\right)=\frac{1}{m !}\left(\mathrm{T}_{\alpha}^{m}\left(2 e^{\sum_{m=0}^{\infty} u_{m}\left(\frac{1}{\alpha} \lambda^{\alpha}\right)^{m}}\right)\right)(\lambda=0)$.

So

$\mathcal{A}_{0}\left(u_{0}\right)=2 e^{u_{0}}$,

$\mathcal{A}_{1}\left(u_{0}, u_{1}\right)=2 u_{1} e^{u_{0}}$,

$\mathcal{A}_{2}\left(u_{0}, u_{1}, u_{2}\right)=\frac{1}{2 !}\left(2\left(2 u_{2} e^{u_{0}}+u_{1}^{2} e^{u_{0}}\right)\right)$

First few terms will be obtained as follows

$u_{1}=\left(\frac{1}{\alpha} x^{\alpha}\right)^{2}$

$u_{2}=\frac{1}{6}\left(\frac{1}{\alpha} x^{\alpha}\right)^{4}$,

$u_{3}=\frac{2}{45}\left(\frac{1}{\alpha} x^{\alpha}\right)^{6}$,

Therefore, the following solution will be obtained

$u(x)=\left(\frac{1}{\alpha} x^{\alpha}\right)^{2}+\frac{1}{6}\left(\frac{1}{\alpha} x^{\alpha}\right)^{4}+\frac{2}{45}\left(\frac{1}{\alpha} x^{\alpha}\right)^{6}+\cdots=$ $-2 \ln \left(\cos \left(\frac{1}{\alpha} x^{\alpha}\right)\right)$

Which is the exact solution.

Example 2 Consider fractional Bratu-type equation with as follows initial condition [15]

$\mathrm{T}_{\alpha}^{2}(u)(x)-\pi^{2} e^{u(x)}=0, \quad 0<\alpha \leq 1$,

$0 \leq x<1, \quad u(0)=0, \mathrm{~T}_{\alpha}(u)(0)=\pi$.

The exact solution of Eq. (13) is $u(x)=-\ln \left(1-\sin \left(\frac{\pi}{\alpha} x^{\alpha}\right)\right.$. 
By using $\mathrm{I}_{\alpha}^{2}=\int_{0}^{x} \int_{0}^{t_{1}}(.) d t_{2} d t_{1}$, we obtain:

$u(x)=u(0)+\left(T_{\alpha} u\right)(0)\left(\frac{1}{\alpha} x^{\alpha}\right)+I_{\alpha}^{2}\left(\pi^{2} e^{u(x)}\right)$.

By substituting $u(x)=\sum_{m=0}^{\infty} u_{m}$, into this equation, we get

$\sum_{m=0}^{\infty} u_{m}=\pi \frac{1}{\alpha} x^{\alpha}+\sum_{m=0}^{\infty} I_{\alpha}^{2}\left(\mathcal{A}_{m}\left(u_{0}, u_{1}, \ldots, u_{m}\right)\right.$,

So, the Adomian scheme would be as follows

$u_{0}(x)=\pi \frac{1}{\alpha} x^{\alpha}, u_{m+1}(x)=\mathrm{I}_{\alpha}^{2}\left(\mathcal{A}_{m}\left(u_{0}, u_{1}, \ldots, u_{m}\right)\right), \quad m=$ $0,1,2, \ldots$

Where

$\mathcal{A}_{0}\left(u_{0}\right)=\pi^{2} e^{u_{0}}$,

$\mathcal{A}_{1}\left(u_{0}, u_{1}\right)=\pi^{2} u_{1} e^{u_{0}}$,

$\mathcal{A}_{2}\left(u_{0}, u_{1}, u_{2}\right)=\frac{1}{2 !}\left(\pi^{2}\left(2 u_{2} e^{u_{0}}+u_{1}^{2} e^{u_{0}}\right)\right)$,

Therefore, the following results will be derived

$u_{1}=e^{\pi\left(\frac{1}{\alpha} x^{\alpha}\right)}-\pi\left(\frac{1}{\alpha} x^{\alpha}\right)-1$,

$u_{2}=-\frac{5}{4}-\frac{\pi}{2}\left(\frac{1}{\alpha} x^{\alpha}\right)+\frac{1}{4} e^{2 \pi\left(\frac{1}{\alpha} x^{\alpha}\right)}-\pi\left(\frac{1}{\alpha} x^{\alpha}\right) e^{\pi\left(\frac{1}{\alpha} x^{\alpha}\right)}+e^{\pi\left(\frac{1}{\alpha} x^{\alpha}\right)}$,

$u_{3}=-\frac{11}{6}+\frac{\pi^{2}}{2}\left(\frac{1}{\alpha} x^{\alpha}\right)^{2} e^{\pi\left(\frac{1}{\alpha} x^{\alpha}\right)}-\frac{\pi}{2}\left(\frac{1}{\alpha} x^{\alpha}\right) e^{2 \pi\left(\frac{1}{\alpha} x^{\alpha}\right)}-\frac{3 \pi}{2} e^{\pi\left(\frac{1}{\alpha} x^{\alpha}\right)}$

$-\frac{\pi}{2}\left(\frac{1}{\alpha} x^{\alpha}\right)+\frac{1}{2} e^{2 \pi\left(\frac{1}{\alpha} x^{\alpha}\right)}+\frac{5}{4} e^{\pi\left(\frac{1}{\alpha} x^{\alpha}\right)}+\frac{1}{12} e^{3 \pi\left(\frac{1}{\alpha} x^{\alpha}\right)}$,

$\vdots$

Four-terms approximation to the solution will be obtained as the following form

$u(x) \approx-\frac{49}{12}-\pi\left(\frac{1}{\alpha} x^{\alpha}\right)+\frac{3}{4} e^{2 \pi\left(\frac{1}{\alpha} x^{\alpha}\right)}-\frac{5 \pi}{2}\left(\frac{1}{\alpha} x^{\alpha}\right) e^{\pi\left(\frac{1}{\alpha} x^{\alpha}\right)}+$ $\frac{13}{4} e^{\pi\left(\frac{1}{\alpha} x^{\alpha}\right)}$

$+\frac{\pi^{2}}{2}\left(\frac{1}{\alpha} x^{\alpha}\right)^{2} e^{\pi\left(\frac{1}{\alpha} x^{\alpha}\right)}-\frac{\pi}{2}\left(\frac{1}{\alpha} x^{\alpha}\right) e^{2 \pi\left(\frac{1}{\alpha} x^{\alpha}\right)}+\frac{1}{12} e^{3 \pi\left(\frac{1}{\alpha} x^{\alpha}\right)}$

In Figure 1, the exact and approximate solutions of fractional Bratu-type equation for $\alpha=0.5$, up to 1.0 , is plotted, It can be concluded from the result that whatever $\alpha$ is closer to 1 , the convergence as well as the accuracy of approximate solution will be better.
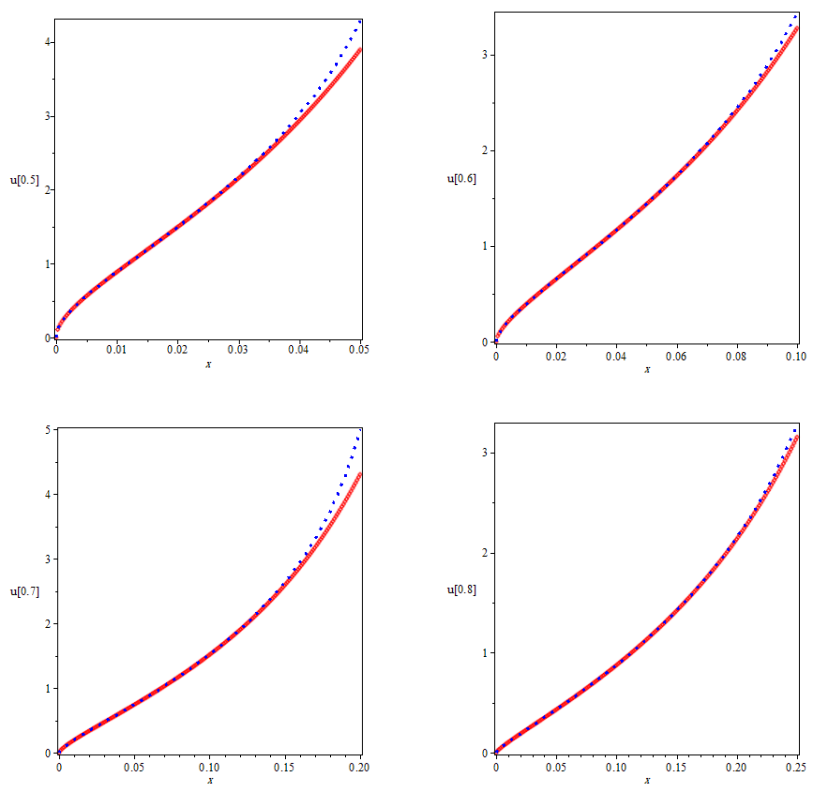

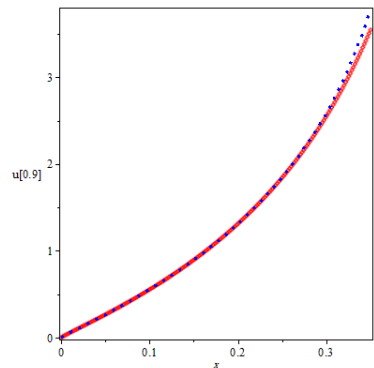

(exact solution (------),

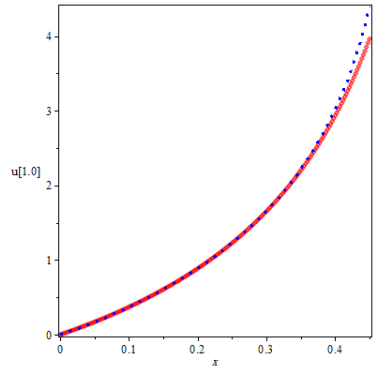

ADM solution $(\ldots .)$.
Fig. 1: The Comparison 4th-Order Approximation of ADM and Exact Solution for Example 2.

Example 3 Consider fractional Bratu-type equation [15]

$\mathrm{T}_{\alpha}^{2}(u)(x)+\pi^{2} e^{-u(x)}=0,0<\alpha \leq 1,0 \leq x<1$,

With initial condition $u(0)=0, \mathrm{~T}_{\alpha}(u)(0)=\pi$.

The exact solution of Eq. (14) is $u(x)=\ln \left(1+\sin \left(\frac{\pi}{\alpha} x^{\alpha}\right)\right.$.

By using Eq. (7), Eq. (13) can be written as follows:

$u(x)=u(0)+\left(T_{\alpha} u\right)(0)\left(\frac{1}{\alpha} x^{\alpha}\right)-I_{\alpha}^{2}\left(\pi^{2} e^{-u(x)}\right)$.

By substituting $u(x)=\sum_{m=0}^{\infty} u_{m}$, we get

$u_{0}(x)=\pi \frac{1}{\alpha} x^{\alpha}$

$u_{m+1}(x)=-\mathrm{I}_{\alpha}^{2}\left(\mathcal{A}_{m}\left(u_{0}, u_{1}, \ldots, u_{m}\right)\right), m=0,1,2, \ldots$

Where Adomian polynomial will be obtained as the following form

$\mathcal{A}_{0}\left(u_{0}\right)=\pi^{2} e^{-u_{0}}$,

$\mathcal{A}_{1}\left(u_{0}, u_{1}\right)=-\pi^{2} u_{1} e^{-u_{0}}$

$\mathcal{A}_{2}\left(u_{0}, u_{1}, u_{2}\right)=\frac{1}{2 !}\left(\pi^{2}\left(-2 u_{2} e^{-u_{0}}+u_{1}^{2} e^{-u_{0}}\right)\right)$,

So, we have

$u_{1}=-e^{-\pi\left(\frac{1}{\alpha} x^{\alpha}\right)}-\pi\left(\frac{1}{\alpha} x^{\alpha}\right)+1$, 
$u_{2}=\frac{5}{4}-\frac{\pi}{2}\left(\frac{1}{\alpha} x^{\alpha}\right)-\frac{1}{4} e^{-2 \pi\left(\frac{1}{\alpha} x^{\alpha}\right)}-\pi\left(\frac{1}{\alpha} x^{\alpha}\right) e^{-\pi\left(\frac{1}{\alpha} x^{\alpha}\right)}-e^{-\pi\left(\frac{1}{\alpha} x^{\alpha}\right)}$

$u_{3}=\frac{11}{6}-\frac{\pi^{2}}{2}\left(\frac{1}{\alpha} x^{\alpha}\right)^{2} e^{-\pi\left(\frac{1}{\alpha} x^{\alpha}\right)}-\frac{\pi}{2}\left(\frac{1}{\alpha} x^{\alpha}\right)-\frac{1}{2} e^{-2 \pi\left(\frac{1}{\alpha} x^{\alpha}\right)}-$

$\frac{3 \pi}{2}\left(\frac{1}{\alpha} x^{\alpha}\right) e^{2 \pi\left(\frac{1}{\alpha} x^{\alpha}\right)}$

$-\frac{\pi}{2}\left(\frac{1}{\alpha} x^{\alpha}\right) e^{-2 \pi\left(\frac{1}{\alpha} x^{\alpha}\right)}-\frac{5}{4} e^{-\pi\left(\frac{1}{\alpha} x^{\alpha}\right)}-\frac{1}{12} e^{-2 \pi\left(\frac{1}{\alpha} x^{\alpha}\right),}$

$\vdots$

Consciously, four-terms approximations to the solution will be as follows:

$$
\begin{aligned}
& u(x) \approx \frac{49}{12}-\frac{\pi}{2}\left(\frac{1}{\alpha} x^{\alpha}\right)-2 e^{-\pi\left(\frac{1}{\alpha} x^{\alpha}\right)}-\pi\left(\frac{1}{\alpha} x^{\alpha}\right) e^{-\pi\left(\frac{1}{\alpha} x^{\alpha}\right)}- \\
& \frac{1}{4} e^{-2 \pi\left(\frac{1}{\alpha} x^{\alpha}\right)} \\
& -\frac{\pi^{2}}{2}\left(\frac{1}{\alpha} x^{\alpha}\right)^{2} e^{-\pi\left(\frac{1}{\alpha} x^{\alpha}\right)}-\frac{\pi}{2}\left(\frac{1}{\alpha} x^{\alpha}\right)-\frac{1}{2} e^{-2 \pi\left(\frac{1}{\alpha} x^{\alpha}\right)}- \\
& \frac{3 \pi}{2}\left(\frac{1}{\alpha} x^{\alpha}\right) e^{2 \pi\left(\frac{1}{\alpha} x^{\alpha}\right)} \\
& -\frac{\pi}{2}\left(\frac{1}{\alpha} x^{\alpha}\right) e^{-2 \pi\left(\frac{1}{\alpha} x^{\alpha}\right)}-\frac{5}{4} e^{-\pi\left(\frac{1}{\alpha} x^{\alpha}\right)}-\frac{1}{12} e^{-2 \pi\left(\frac{1}{\alpha} x^{\alpha}\right)} .
\end{aligned}
$$

Figures 2 confirmed previous results.
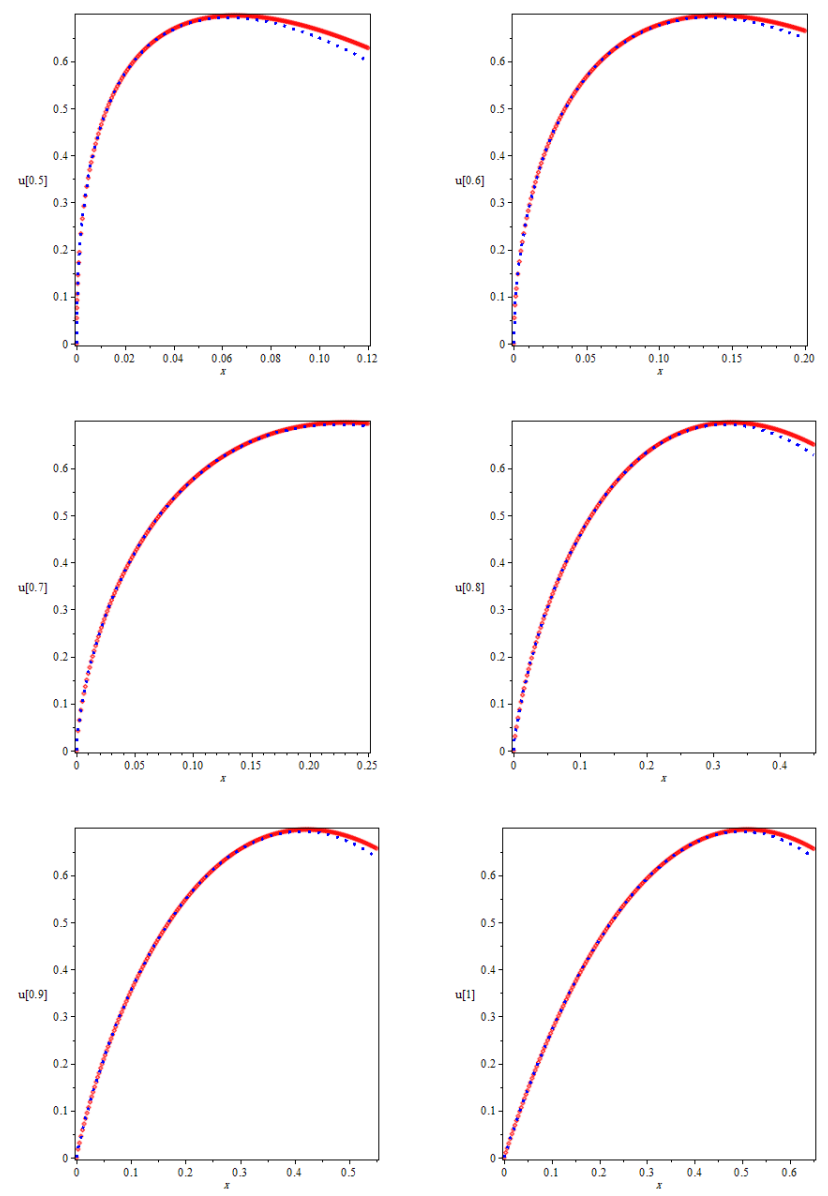

(exact solution (-----),

ADM solution (.....))

Fig. 2: The Comparison 4th-Order Approximation of ADM and Exact Solution for Example 3.

\section{Conclusion}

In this paper, Adomian Decomposition method has been applied to obtain the solutions of fractional differential equations. To this aim, a conformable fractional derivative has been used to find the solution. The results showed that the definition is the simplest tool to obtain the approximation solutions of nonlinear fractional differential equations in comparison to the other definitions. To show the effectiveness and simplicity of the method, fractional Bratutype equations as an example have been solved with form conformable fractional derivative and Adomian Decomposition method.

\section{References}

[1] R. Khalil, M. A. Horani, A. Yousef and M. Sababheh, A new definition of fractional derivative, Journal of Computational and Applied Mathematics, $264 \quad$ (2014) https://doi.org/10.1016/j.cam.2014.01.002.

[2] T. Abdeljawad, on conformable fractional calculus, Journal of Computational and Applied Mathematics, 279 (2015) 57-66 https://doi.org/10.1016/j.cam.2014.10.016.

[3] F.B.M. Duarte, J. A. Tenreiro Machado, Chaotic phenomena and fractional- order dynamics in the trajectory control of redundant manipulators, Nonlinear Dynamics, 29 (2002) 342-362. https://doi.org/10.1023/A:1016559314798.

[4] O. P. Agrawal, A general formulation and solution scheme for fractional optimal control problems, Nonlinear Dynamics, 34 (2004) 323-337. https://doi.org/10.1007/s11071-004-3764-6.

[5] N. Engheta, On fractional calculus and fractional multipoles in electromagnetism, IEEE Transactions on Antennas and Propagation, 44 (1996) 554-566. https://doi.org/10.1109/8.489308.

[6] R. L. Magin, Fractional calculus models of complex dynamics in biological tissues, Computer and Mathematics with Applications, 59 (2010) 1586-1593. https://doi.org/10.1016/j.camwa.2009.08.039

[7] V. V. Kulish, Jos L. Larg, Application of fractional calculus to fluid mechanics, Journal of Fluids Engineering, 134 (2002).

[8] K. B. Oldhom, Fractional differential equations in electrochemistry, Advances in Engineering Software, 41(2010) 9-12. https://doi.org/10.1016/j.advengsoft.2008.12.012.

[9] V. Gafiychuk, B. Datsko, V. Meleshko, Mathematical modeling of time fractional reaction diffusion systems, Journal of Computational and Applied Mathematics, 220 (2008) 215-225. https://doi.org/10.1016/j.cam.2007.08.011.

[10] Seadawy A. R, Stability analysis solutions for nonlinear threedimensional modified Korteweg-de Vries-Zakharov-Kuznetsov equation in a magnetized electron-positron plasma, Physica A: Statistical Mechanics and its Applications, 455 (2016) 44-51. https://doi.org/10.1016/j.physa.2016.02.061.

[11] F. C. Meral, T. J. Royston, R. Magin, Fractional calculus in viscoelasticity:an experimental study, Communications in Nonlinear Science and Numerical Simulation, 15 (2010) 939-945. https://doi.org/10.1016/j.cnsns.2009.05.004.

[12] F. Mainardi, Fractional calculus: some basic problem in continuum and statistical mechanics, in: A. Carplnteri, F. Mainardi (Eds.), Fractals and Fractional calculus in continuum Mechanics, SpringerVerlag, New York, (1997) 291-348. https://doi.org/10.1007/978-37091-2664-67.

[13] A. R. Seadawy, Nonlinear wave solutions of three-dimensional Zakharov-Kuznetsov-Burgers equation in dusty plasma, Physica A: Statistical Mechanics and its Applications, 439 (2015) 124-131. https://doi.org/10.1016/j.physa.2015.07.025.

[14] V. Daftardar-Gejji, H. Jafari, Solving a multi- order fractional differential equation using Adomian Decomposition, Applied Mathematics and Computation,189 (2007) 541-548. https://doi.org/10.1016/j.amc.2006.11.129.

[15] B. Ghazanfari, A. Sepahvandzadeh, Adomian decomposition method for solving fractional Bratu-type equations, Journal of mathematics and computer science, 8 (2014) 236-244.

[16] O. Abdulaziz, I. Hashim, S. Momani, solving systems of fractional differential equations by homotopy perturbation method, Physics $\begin{array}{llll}\text { Letters } & \text { A, } & 372 & \text { (2008) 451-459. }\end{array}$ https://doi.org/10.1016/j.physleta.2007.07.059.

[17] B. Ghazanfari, A. G. Ghazanfari, M. Fuladvand, Modification of the homotopy perturbation method for numerical solution of Nonlinear Wave and of Nonlinear Wave Equations, Journal of mathematics and computer science, 3 (2011) 212-224.

[18] M. Mahmoudi, M. V. Kazemi, Solving singular BVPs Ordinary Differential Equations by Modified Homotopy Perturbation Method, Journal of mathematics and computer science, 7 (2013) 138-143.

[19] M. Rabbani, New Homotopy Perturbation Method to Solve NonLinear Problems, Journal of mathematics and computer science, 7 (2013) 272-275. 
[20] I. Hashim, O. Abdulaziz, S. Momani, Homotopy Analysis Method for fractional IVPs, Communications in Nonlinear Science and Numerical Simulation, $14 \quad$ (2009) 674-684 https://doi.org/10.1016/j.cnsns.2007.09.014.

[21] G. Wu, E. W. M. Lee, Fractional variational iteration method and its application, Physics Letters A, 374 (2010) 2506-2509. https://doi.org/10.1016/j.physleta.2010.04.034.

[22] Z. Odibat, S. Momani, V. Suat Erturk, Generalized differential transform method: application to differential equations of fractional order, Applied Mathematics and Computation, 197 (2008) 467-477. https://doi.org/10.1016/j.amc.2007.07.068.

[23] Y. Zhang, A finite difference method for fractional partial differential equation, Applied Mathematics and Computation, 215 (2009) 524-529. https://doi.org/10.1016/j.amc.2009.05.018.

[24] H. Azizi, Gh. Barid Loghmani A numerical method for space fractional diffusion equations using a semi-disrete scheme and Chebyshev collocation method, Journal of mathematics and computer science, 8 (2014) 226-235.

[25] A. Neamaty, B. Agheli, R. Darzi, Solving Fractional Partial Differential Equation by Using Wavelet Operational Method, Journal of mathematics and computer science, 7 (2013) 230-240.

[26] Y. Aksoy, M. Pakdemirli, New perturbation-iteration solutions for Bratu-type equations, Computers and Mathematics with Applica$\begin{array}{llll}\text { tions, } & 59 & \text { (8) } & \text { (2010) }\end{array}$ https://doi.org/10.1016/j.camwa.2010.01.050

[27] M. Ilie, J. Biazar, Z. Ayati, General solution of Bernoulli and Riccati fractional differential equations based on conformable fractional derivative, International Journal of Applied Mathematical Research, 6(2) (2017) 49-51. https://doi.org/10.14419/ijamr.v6i2.7014.

[28] M. Ilie, J. Biazar, Z. Ayati, Application of the Lie Symmetry Analysis for second-order fractional differential equations, Iranian Journal of Optimization, 9(2) (2017) 79-83.

[29] M. Ilie, J. Biazar, Z. Ayati, The first integral method for solving some conformable fractional differential equations, Optical and $\begin{array}{llll}\text { Quantum } & \text { Electronics, } & \text { Vol.50 (2018) } & \text { No.2 }\end{array}$ https://doi.org/10.1007/s11082-017-1307-x.

[30] G. Adomian, Solving Frontier Problems of Physics: The Decomposition Method, Kluwer Academic Publishers, Boston, (1994). https://doi.org/10.1007/978-94-015-8289-6.

[31] G. Adomian, A review of the decomposition method in applied mathematics, Journal of Mathematical Analysis and Applications, 135 (1988) 501-544. https://doi.org/10.1016/0022-247X(88)901709.

[32] J. Biazar, E. Babolian, A. Nouri, R. Islam, An alternate algorithm for computing Adomian decomposition method in special cases, Applied Mathematics and Computation, 138 (2-3) (2003) 523-529. https://doi.org/10.1016/S0096-3003(02)00174-1.

[33] J. Biazar, M. Ilie, A. Khoshkenar, An improvement to an alternate algorithm for computing Adomian polynomials in special cases, Applied Mathematics and Computation, 173(1) (2006) 582-592. https://doi.org/10.1016/j.amc.2005.04.052. 